$12-2011$

\title{
Accelerated Fatigue Crack Growth Simulation in a Bimaterial Interface
}

\author{
R. Moslemian \\ Technical University of Denmark \\ Anette M. Karlsson \\ Cleveland State University, a.karlsson@csuohio.edu \\ C. Berggreen \\ Technical University of Denmark
}

Follow this and additional works at: https://engagedscholarship.csuohio.edu/enme_facpub

Part of the Mechanical Engineering Commons

How does access to this work benefit you? Let us know!

\section{Publisher's Statement}

NOTICE: this is the author's version of a work that was accepted for publication in International Journal of Fatigue. Changes resulting from the publishing process, such as peer review, editing, corrections, structural formatting, and other quality control mechanisms may not be reflected in this document. Changes may have been made to this work since it was submitted for publication. A definitive version was subsequently published in International Journal of Fatigue, 33, 12, (12-01-2011); 10.1016/j.ijfatigue.2011.06.006

\section{Original Citation}

Moslemian, R., Karlsson, A. M., and Berggreen, C., 2011, "Accelerated Fatigue Crack Growth Simulation in a Bimaterial Interface," International Journal of Fatigue, 33(12) pp. 1526-1532.

This Article is brought to you for free and open access by the Mechanical Engineering Department at EngagedScholarship@CSU. It has been accepted for inclusion in Mechanical Engineering Faculty Publications by an authorized administrator of EngagedScholarship@CSU. For more information, please contact library.es@csuohio.edu. 


\title{
Accelerated fatigue crack growth simulation in a bimaterial interface
}

\author{
R. Moslemian ${ }^{\text {a }}$, A.M. Karlsson ${ }^{\text {b }}$, C. Berggreen ${ }^{\mathrm{a}, *}$ \\ ${ }^{a}$ Department of Mechanical Engineering. Technical University of Denmark, Nils Koppels Allé, Building 403, DK-2800 Kgs. Lyngby, Denmark \\ ${ }^{b}$ Department of Mechanical Engineering, University of Delaware, Newark, DE 19716, United States
}

\section{Introduction}

Interface fatigue crack growth is one of the most critical damages that layered structures, such as monolithic fiber reinforced or sandwich composites, can experience. Design against fatigue failure of these types of structures is associated with many challenges due to the complexity of the interface fracture problem. Only a limited number of studies on the interface fatigue crack growth have been reported in the literature [1-3]. Shipsha and co-authors [1] determined the crack growth rate in the interface of a sandwich beam under global mode I and II loading experimentally [1]. Quispitupa and Shafiq [2] conducted fatigue tests of sandwich beams via three-point bending. They observed both global mode I and mode II cracking in the face/core interface of the specimens [2]. Berkowitz and Johnson [3] performed fatigue tests of a modified double cantilever beam (DCB) [3]. They used the compliance of the DCB specimen to determine the crack length and the crack growth rate. They also studied the temperature effect on the crack propagation in a particular sandwich system, and elucidated the significant effect of temperature on the crack propagation rate in a sandwich face/core interface [3].

* Corresponding author. Tel.: +45 45251 373; fax: +45 45884355 .

E-mail addresses: rmo@mek.dtu.dk (R. Moslemian), karlsson@udel.edu (A.M. Karlsson), cbe@mek.dtu.dk (C. Berggreen).

URLs: http://me.udel.edu (A.M. Karlsson), http://www.mek.dtu.dk (C. Berggreen).
To assess the damage tolerance of a layered structure exposed to cyclic loading (fatigue), experiments are typically conducted on both intact specimens and on specimens with a pre-existing (known) crack. This requires special testing facilities and is usually very costly and time consuming. Due to the difficulties and expense associated with conducting fatigue experiments, considerable efforts have been directed in recent years into simulating fatigue crack growth using numerical methods. Maziere and Fedelich [4] simulated 2D fatigue crack propagation using the finite element method and implementation of the strip-yield model. Their model assumes that, at each cycle, the crack growth results from the variation of the crack tip opening displacement (CTOD). They used cohesive elements with linear-elastic, perfectly-plastic behavior to simulate crack growth [4]. Kiyak and co-authors [5] simulated fatigue crack growth under low cycle fatigue at high temperature in a single crystal super alloy. To simulate the crack growth, they implemented a node release technique and released the nodes in each cycle according to an experimentally measured crack growth rate. The simulation results were compared with the experiments on the Single Edge Notch specimens of the $\mathrm{Ni}$ based single crystal superalloy PWA 1483 at $950{ }^{\circ} \mathrm{C}$ results on the basis of the computed crack tip opening displacement (CTOD) [5]. Shi and Zhang [6] simulated the interfacial crack growth of fiber reinforced composites under tension-tension cyclic loading using the finite element method. In their model, the energy release rate is calculated and utilized in Paris law in order to calculate crack growth rate [6]. Ramanujam and co-authors [7] studied the 
fatigue growth of fiber reinforced composite laminates under thermal cyclic loading using combined experimental and computational investigations [7].

In all abovementioned studies, the simulation of fatigue crack growth was limited to only a few cycles due to the need of a high mesh density at the crack tip and subsequently required high computational time. This illustrates the main obstacle confronting any attempt to combine fracture mechanics and the finite element method to simulate fatigue crack growth. The aim of this study is to overcome this obstacle by proposing a method to accelerate finite element fatigue crack growth simulations. To this end, the "cycle jump concept" is employed to shorten the simulations by eliminating the need of simulating all individual cycles. The cycle jump concept can be utilized to estimate the long term degradation of the load carrying capacity of structures evolving over many cycles. The cycle jump concept has mostly been utilized in the context of damage mechanics. Ladeveze and co-authors $[8,9]$ introduced the "Large Time Increments Method" dividing the equations of the initial boundary value problem into linear and nonlinear equations, where the linear equation are global and the nonlinear equations are local in space. They used the global part with extrapolation algorithms to jump over cycles [8,9]. Fish and co-authors $[10,11]$ developed a fatigue model for brittle composite materials where the evolution of fatigue damage is approximated by the first order initial value problem with respect to the number of load cycles [10,11]. Kiewel et al. [12] developed a scheme to extrapolate the complete set of internal variables over a certain range of cycles. They used piecewise polynomials and spline functions for the desired variables on each integration point in a finite element model [12]. Van Paepegem and co-authors [13] adopted the cycle jump method to a set of fatigue loading cycles at arbitrary chosen intervals and determined the effect of the fatigue loading in between. Their extrapolation scheme works based on extrapolation of the damage parameter by using the explicit Euler integration formula [13]. Cojocaru and Karlsson [14] employed the cycle jump technique to simulate the response of Thermal Barrier Coatings (TBCs) under cyclic thermal loading, where the structure evolves due to changing material properties during high temperature [14]. In this case, damage mechanics was not used. They proposed a control function that automatically monitors the length of the cycle jump to ensure a realistic solution [14].

In this work, the method developed by Cojocaru and Karlsson [14] is adopted with some modifications so to take into account the change in the geometry of the finite element model and simulate fatigue crack propagation. Using the developed finite element scheme, fatigue crack propagation in the face/core interface of a sandwich beam is simulated. Results are compared with a reference analysis, simulating all individual cycles, to verify the proposed cycle jump technique.

\section{Cycle jump technique}

In structures subjected to cyclic loading, parameters such as deflection, stress, strain, material properties and/or geometry (for example cracks) typically evolve over time. This evolution results in both global and local changes of the structural behavior, where the global changes correspond to a general long term trend which can be expressed in term of mathematical functions, as suggested by Cojocaru and Karlsson [14]. By utilizing these mathematical functions, extrapolation schemes can be employed to determine the long term response of the structure. Such an extrapolation scheme can be used in numerical simulations to accelerate the analyses and make them computationally effective. In this study, the cycle jump technique utilizing the extrapolation schemed developed by Cojocaru and Karlsson [14] is implemented in a crack propagation finite element routine to simulate bimaterial fatigue crack growth.

The scheme developed in Ref. [14] will be summarized here for completeness of the presentation. First, a set of initial load cycles are simulated using the finite element method and the global evolution function is established for each state variable monitored. This global evolution function is then used to extrapolate the state variable over a number of cycles [14]. The key question here is the accuracy of the extrapolated variables. To examine and control the accuracy of the extrapolation the number of jump cycles is determined through a criterion with a control function [14]. The determined extrapolated state is used as an initial state for additional finite element simulations and next cycle jumps, see Fig. 1 [14].

Assuming that a FE analysis has been conducted for at least three computed load cycles, see Fig. 2, for each state variable monitored, $y=y(t)$, where $t$ is time, the discrete slope can be defined for every two adjacent cycles as [14]

$S_{12}\left(t_{2}\right)=\frac{y\left(t_{2}\right)-y\left(t_{1}\right)}{\Delta t_{c y c}}$

$S_{23}\left(t_{3}\right)=\frac{y\left(t_{3}\right)-y\left(t_{2}\right)}{\Delta t_{c y c}}$

where $\Delta t_{c y c}=t_{2}-t_{1}=t_{3}-t_{2}$ is the time of each cycle. The parameter $q_{y}$ is introduced as the maximum relative error to control the accuracy of the simulation by using the following criterion [14]

$\left|\frac{S_{\text {jump }}\left(t_{3}+\Delta t_{y, j u m p}\right)-S_{23}\left(t_{3}\right)}{S_{23}\left(t_{3}\right)}\right| \leqslant q_{y}$

where $q_{y}$ is the maximum allowed relative error, $\Delta t_{y, j u m p}$ the number of jumped cycles and $S_{\text {jump }}$ is the estimated slope after the jump using linear extrapolation given by [14]

$S_{\text {jump }}\left(t_{3}+\Delta t_{y, j u m p}\right)=S_{23}\left(t_{3}\right)+\frac{S_{23}\left(t_{3}\right)-S_{12}\left(t_{2}\right)}{\Delta t_{c y c}} \Delta t_{y, j u m p}$

The introduced criterion ensures that the slope of the increment of the variable $y$ after the cycle jump is "close enough" to its slope before the jump. $q_{y}$ is specified by the user for each state parameters such as deflection or material properties [14]. From Eqs. (3) and (4) the allowed jump for each extrapolated parameter is determined by [14]

$\Delta t_{y, j u m p}=q_{y} \Delta t_{c y c} \frac{\left|S_{23}\left(t_{3}\right)\right|}{\left|S_{23}\left(t_{3}\right)-S_{12}\left(t_{2}\right)\right|} \leftarrow$

Since the jump is determined for a set of state variables, the allowed jump $\Delta t_{j u m p}$ is chosen as the minimum of the computed allowed jump times for each variable [14]:

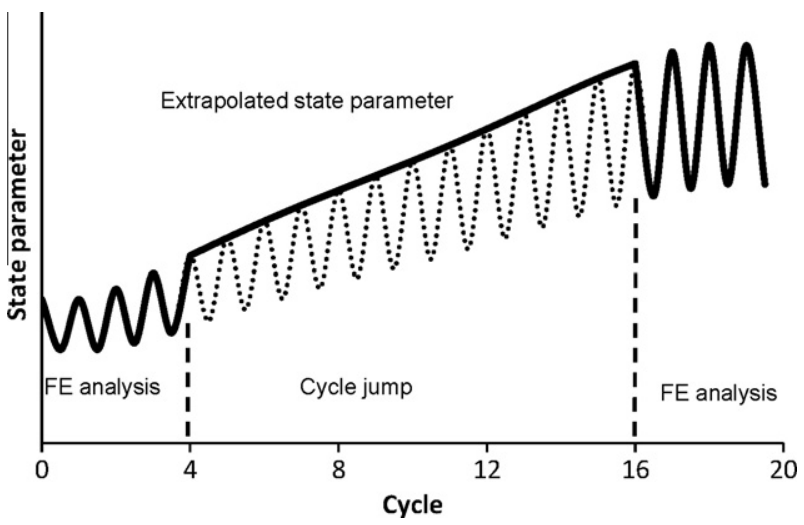

Fig. 1. The schematic representation of the cycle jump technique, after Ref. [14] 


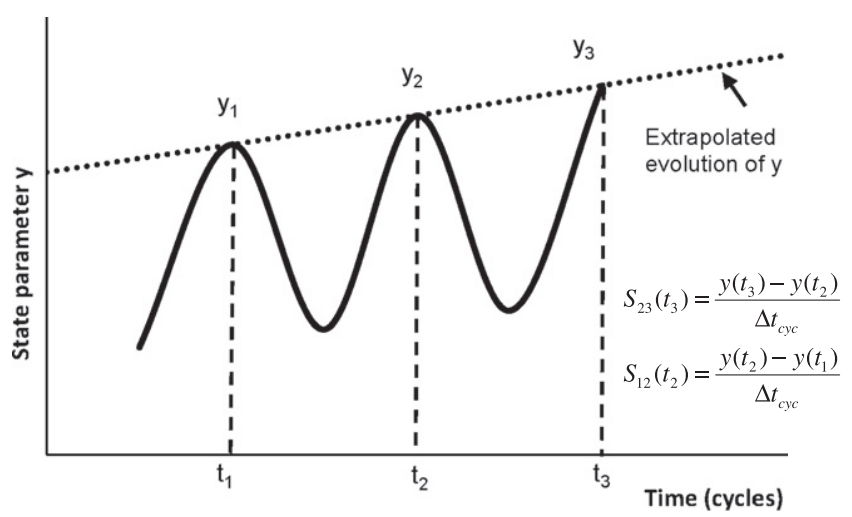

Fig. 2. The schematic representation of the cycle jump technique, after Ref. [14].

$\Delta t_{\text {jump }}=\Delta t_{c y c}\left\lfloor\min \left\{\Delta t_{y, j u m p}\right\} / \Delta t_{c y c}\right\rfloor$

To extrapolate the state variables after each jump the Heun integrator is used as [14]

$y\left(t_{3}+\Delta t_{\text {jump }}\right)=y\left(t_{3}\right)+\frac{1}{2}\left[S\left(23\left(t_{3}\right)+S_{\text {jump }}\left(t_{3}+\Delta t_{\text {jump }}\right)\right] \Delta t_{\text {jump }}\right.$

By substituting Eq. (4) into Eq. (7):

$y\left(t_{3}+\Delta t_{j u m p}\right)=y\left(t_{3}\right)+S_{23}\left(t_{3}\right) \Delta t_{j u m p}$

$$
+\left[S_{23}\left(t_{3}\right)-S_{12}\left(t_{2}\right)\right] \frac{\left(\Delta t_{j u m p}\right)^{2}}{2 \Delta t_{c y c}}
$$

The above extrapolation scheme is most suitable for structures with slowly evolving properties, in a quasi-linear manner. In case of more nonlinear behavior, higher order integrators could be implemented. However, Cojocaru and Karlsson [14] showed that the extrapolation scheme is able to capture highly nonlinear behavior by conducting shorter or no jumps. This of course does not save computational time, but ensure at least an acceptable solution.

\section{Numerical example}

The cycle jump technique described above will now be implemented in a FE-based numerical simulation for investigating fatigue crack propagation in the face/core interface of a sandwich beam. A sandwich structure consists of two strong and stiff face sheets bonded to a core of low density. The face sheets in the sandwich resist in-plane and bending loads. The core separates the face sheets to increase the bending rigidity and strength of the structure, and to transfers shear forces between the face sheets [15]. However, the bonding between the face sheets and core may compromise the benefits of a sandwich structure, if the bonding is not adequate or absent (face/core debond) due to manufacturing flaws, or if damage is inflicted during service. Growth of a face/core interface crack under cyclic loading can results in compromising the overall structural carrying capacity and lifetime of a sandwich structure.

Interface fatigue crack growth in a sandwich beam consisting of $2.8 \mathrm{~mm}$ thick plain weave E-glass/epoxy face sheets over a $50 \mathrm{~mm}$ thick Divinycell H130 PVC foam [16] core is simulated using a commercial finite element code, ANSYS version 11 [17]. Face sheet and

Table 1

Face and core material properties [16]

\begin{tabular}{llll}
\hline Material & $E(\mathrm{MPa})$ & $G(\mathrm{MPa})$ & $v$ \\
\hline Face sheet & 19,400 & 7400 & 0.31 \\
Core: H130 & 170 & 50 & 0.33 \\
\hline
\end{tabular}

core material properties are listed in Table 1 . The length and width of the beam are $215 \mathrm{~mm}$ and $65 \mathrm{~mm}$ respectively. The beam contains an initial face/core crack of $10 \mathrm{~mm}$ length. 8-noded isoparametric elements (PLANE82) are used in the finite element model. The finite element model of the beam is shown in Fig. 3. The strain energy release rate and mode-mixity are calculated from the finite element analysis in the end of each cycle. Strain energy release rate, $G$, and mode-mixity phase angle, $\psi$, are determined from relative nodal pair displacements along the crack flanks obtained from the finite element analysis using the "CSDE method" outlined in Ref. [18]. Unlike homogenous materials in a bimaterial interface mode-mixity is not directly linked to the opening or shearing displacements of the crack flanks or the normal and shear stresses in front of the crack tip, but a distortion exists. The energy release rate and the phase angle are for example given by [19]:

$G=\frac{\pi\left(1+4 \varepsilon^{2}\right)}{8 H_{11} x}\left(\frac{H_{11}}{H_{22}} \delta_{y}^{2}+\delta_{x}^{2}\right)$

$\left.\Psi=\tan ^{-1} \sqrt{\frac{H_{22}}{H_{11}}} \frac{\delta_{x}}{\delta y}\right)\left(-\varepsilon \ln \left(\frac{x}{h}\right)+\tan ^{-1}(2 \varepsilon)\right.$

where $\delta_{y}$ and $\delta_{x}$ are the opening and sliding relative displacement of the crack flanks; $h$ is the characteristic length of the crack problem and has no direct physical meaning, see [19]. Thus, it is here arbitrarily chosen as the face sheet thickness. The basic assumption of the Eqs. (9) and (10) is that the sandwich interface is bimaterial, a more detailed analysis of sandwich interface as a tri-material can be found in [20]. $H_{11}$ and $H_{22}$, are bimaterial constants, depending on material compliances [19]:

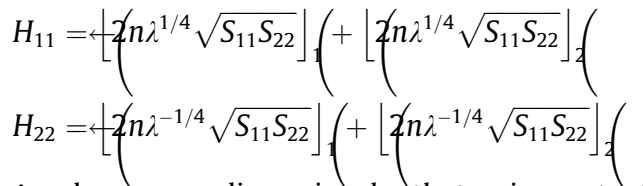

$\lambda$ and $n$ are non-dimensional orthotropic constants given in terms of the elements $S_{11}$ and $S_{22}$ of the compliance matrix:

$\lambda=\frac{S_{11}}{S_{22}}$

$n=\sqrt{\frac{1}{2}(1+\rho)}\left(-\rho=\frac{1}{2} \frac{2 S_{12}+S_{66}}{\sqrt{S_{11} S_{22}}}\right.$

The compliance elements for plane stress conditions are given by

$S_{11}=\frac{1}{E_{1}} \quad S_{12}=S_{21}=-\frac{v_{12}}{E_{1}}=-\frac{v_{21}}{E_{2}}$

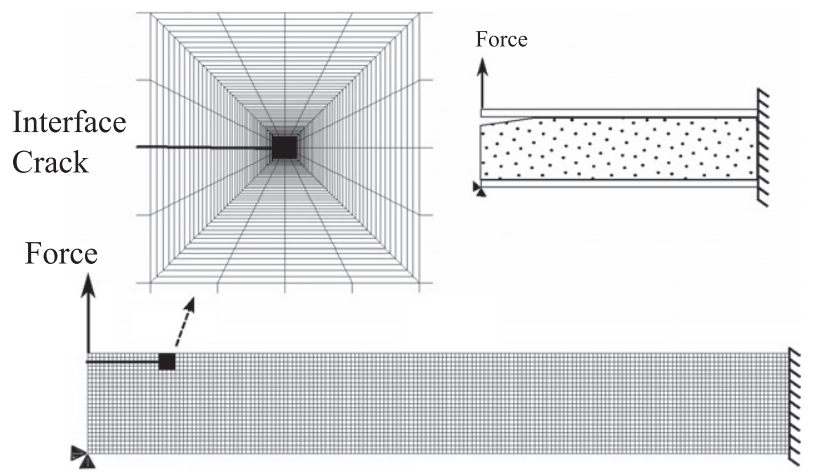

Fig. 3. Finite element model of the sandwich beam. 
$S_{22}=\frac{1}{E_{2}} \quad S_{66}=\frac{1}{G_{12}}$

For plane strain conditions,

$S_{i j}^{* \leftarrow}=S_{i j}-\frac{S_{i 3} S_{j 3}}{S_{33}}$

The oscillatory index, $\varepsilon$, in Eqs. (9) and (10) is given as

$\varepsilon=\frac{1}{2 \pi} \ln \left(\frac{1-\beta}{1+\beta}\right)$

where

$\beta=\frac{\left[S_{12}+\sqrt{S_{11} S_{22}}\right]_{2}-\left[S / 12+\sqrt{S_{11} S_{22}}\right]_{1}}{\sqrt{H_{11} H_{2}}}$

The strain energy release rate and the mode-mixity phase angle are used as the two state variables for the extrapolation and cycle jump in the cycle jump technique. These two parameters are selected since they are the only required parameters for determination of the crack growth length.

Utilizing the relationships between crack growth rate vs. strain energy release rate for a range of mode-mixities as inputs to the FE routine, the crack increment for each cycle is determined and the finite element model with a new crack length is updated. A remeshing algorithm is employed to simulate the crack growth. Due to the current lack of suitable experimental fatigue crack growth rate data, the crack growth rate vs. strain energy release rate relation is assumed linear. This assumption has been made for the simplicity of the problem, for more information see [21]. For mode-mixity phase angles larger and smaller than $10^{\circ}$ fatigue crack growth rate is chosen arbitrarily as

$$
\begin{aligned}
& \frac{d a}{d N}=0.001 \Delta G \quad \text { for } \Psi>-10^{\circ} \\
& \frac{d a}{d N}=0.0008 \Delta G \quad \text { for } \Psi<-10^{\circ}
\end{aligned}
$$

where $\Delta G=G_{\max }-G_{\min }$ is the difference between maximum and minimum strain energy release rate in each cycle and $d a / d N$ is the crack growth rate. The simulation is conducted using force control with maximum amplitude of $0.45 \mathrm{kN}$ and loading ratio of 0.1 .

Fig. 4 shows a route diagram for the implementation of the fatigue crack growth and cycle jump routines.

\section{Results and discussions}

Fig. 5a and $b$ shows the strain energy release rate and phase angle diagrams as a function of the crack length obtained from the numerical simulations of the analyzed debonded sandwich beam at the maximum loading amplitude. The energy release rate increases with increasing crack length up to $60 \mathrm{~mm}$ and then decreases. This can be attributed to the increasing membrane forces as the crack length increases. In the first cycles with increasing crack length, because of small membrane forces the deflection at the crack tip increases, resulting in higher strain energy release rate. However, as the crack length increases, the membrane forces increases and a bigger part of the total strain energy in the specimen goes into stretching of the debonded face sheet rather than creating new crack surfaces, resulting in a decreasing energy release rate at the crack tip. Fig. 5b shows that the phase angle increases with increasing crack length showing that the crack tip loading is more mode II dominant at larger crack lengths. The negative phase angle shows the tendency of the crack to kind towards the face sheet [19].

The fatigue crack propagation simulation was conducted on the sandwich beam for 500 cycles. To study the effect of the control parameter on the accuracy and speed of the simulation, simula-

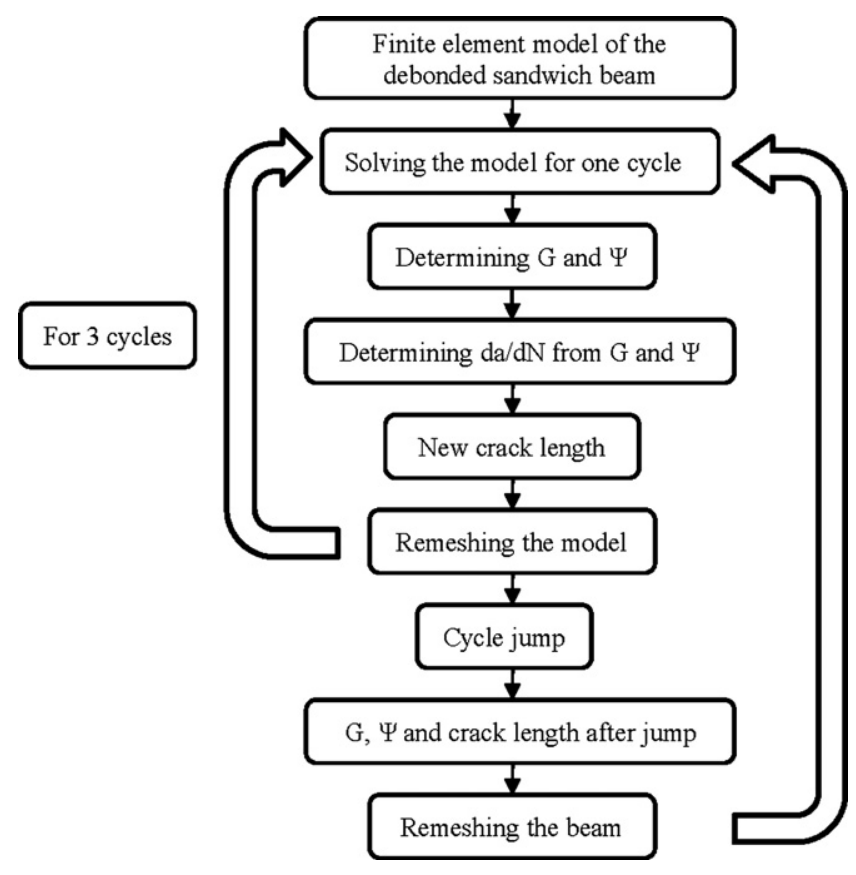

Fig. 4. The route diagram of the implementation of the fatigue crack growth and cycle jump routines.

tions with different control parameters, $q_{y}$, were conducted. A reference simulation, simulating all individual cycles was performed to verify the accuracy of the simulations using the cycle jump method. Fig. $6 \mathrm{a}$ and $\mathrm{b}$ shows the deflection of the loading point ("Y deflection") as a function cycles for two different control parameters $q_{G}=q_{\Psi}=0.05$ and $q_{G}=q_{\Psi}=0.2$.

More cycles are needed in the simulation with a smaller control parameter $q_{G}=q_{\Psi}=0.05$ as expected, but the calculated deflections show a good agreement with the reference analysis. When the control parameter is increased to $q_{G}=q_{\Psi}=0.2$ fewer simulated cycles are needed, but as it can be seen in Fig. 6b, the deflection of the debonded face sheet in the simulation using the cycle jump technique is lower than the reference simulation showing inaccuracy of the simulation. Fig. 7 shows $\Delta G$ vs. the number of cycles. Even though $\Delta G$ shows a highly nonlinear behavior, the cycle jump technique is able to capture this behavior by conducting small or no jumps. In the simulation with the control parameter $q_{G}=q_{\Psi}=0.05$ a fair agreement, see Fig. 7a, between the reference analysis and simulation using the cycle jump technique can be seen. However the results from the simulation with a control parameter $q_{G}=q_{\Psi}=0.2$ show some inaccuracies, see Fig. 7b.

Crack length vs. cycles diagrams for two control parameters $q_{G}=q_{\Psi}=0.05$ and $q_{G}=q_{\Psi}=0.2$ are shown in Fig. 8. In the initial cycles (up to 200 cycles) because of a high growth rate of $\Delta G$ (see Fig. 7), the crack growth rate is large but approaching the end of 500 cycles with decreasing $\Delta G$, crack increment becomes smaller. The simulation with $q_{G}=q_{\Psi}=0.05$ follows the reference simulation with good agreement, but the simulation with $q_{G}=q_{\Psi}=0.2$ shows again less accuracy.

Fig. 9 shows the phase angle vs. number of cycles. The same conclusion can be drawn upon the accuracy of the simulation using cycle jump method and the two control parameters $q_{G}=q_{\Psi}=0.05$ and $q_{G}=q_{\Psi}=0.2$.

To measure the computational efficiency of the cycle jump technique for the analyses with different control parameters, the ratio $R$ is introduced [14]:

$R=\frac{N_{\text {jump }}}{N_{\text {ref }}}$ 

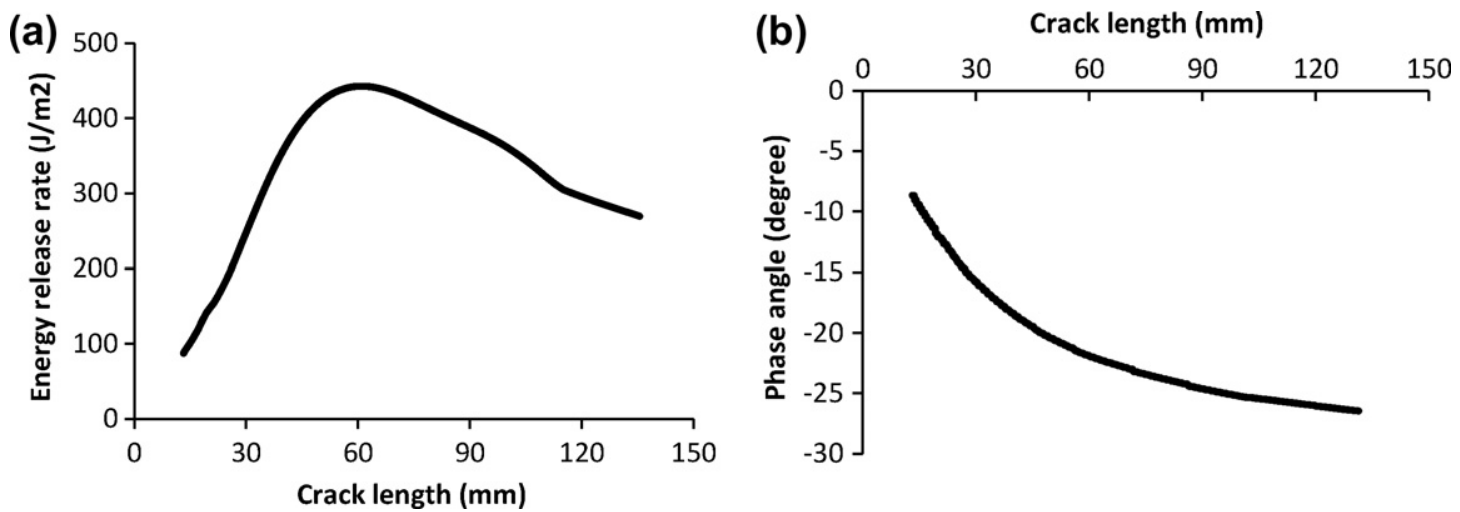

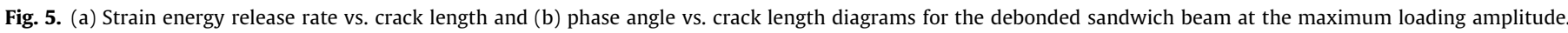
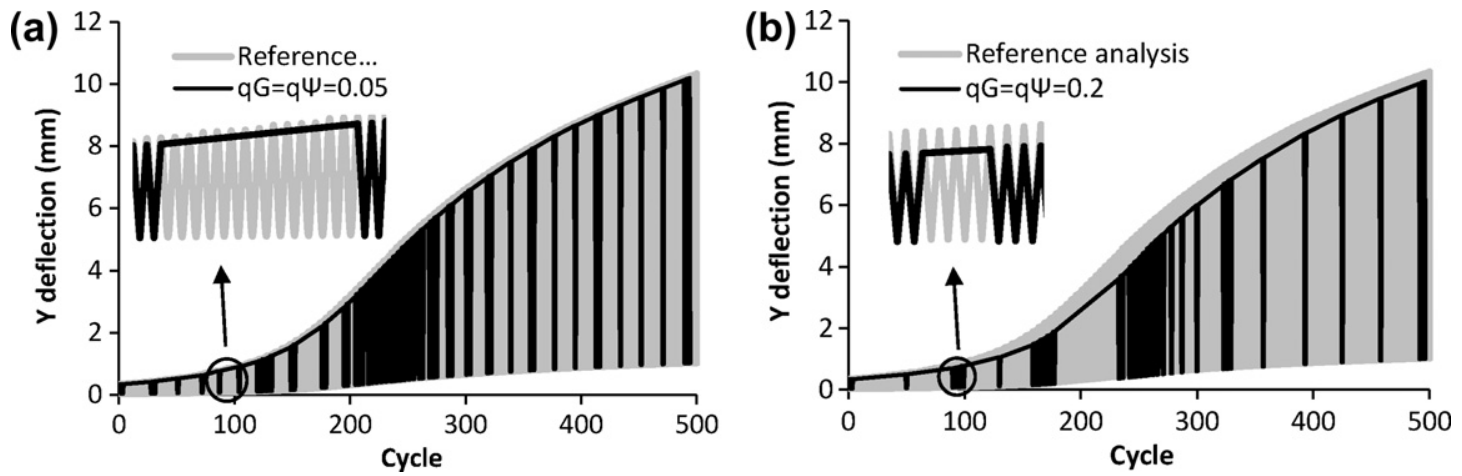

Fig. 6. Deflection of the face sheet at the point of loading (Y deflection) vs. number of cycles for (a) control parameter $q_{G}=q_{\Psi}=0.05$ and (b) $q_{G}=q_{\Psi}=0.2$.
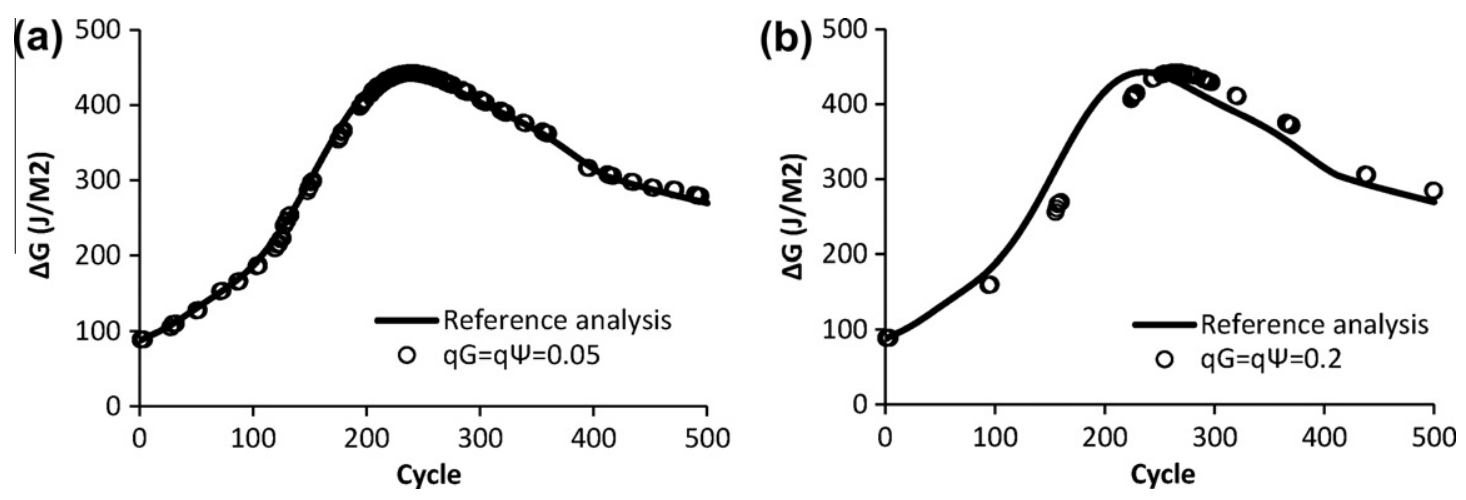

Fig. 7. $\Delta G$ at the crack tip vs. cycles for (a) control parameter $q_{G}=q_{\Psi}=0.05$ and (b) $q_{G}=q_{\Psi}=0.2$.
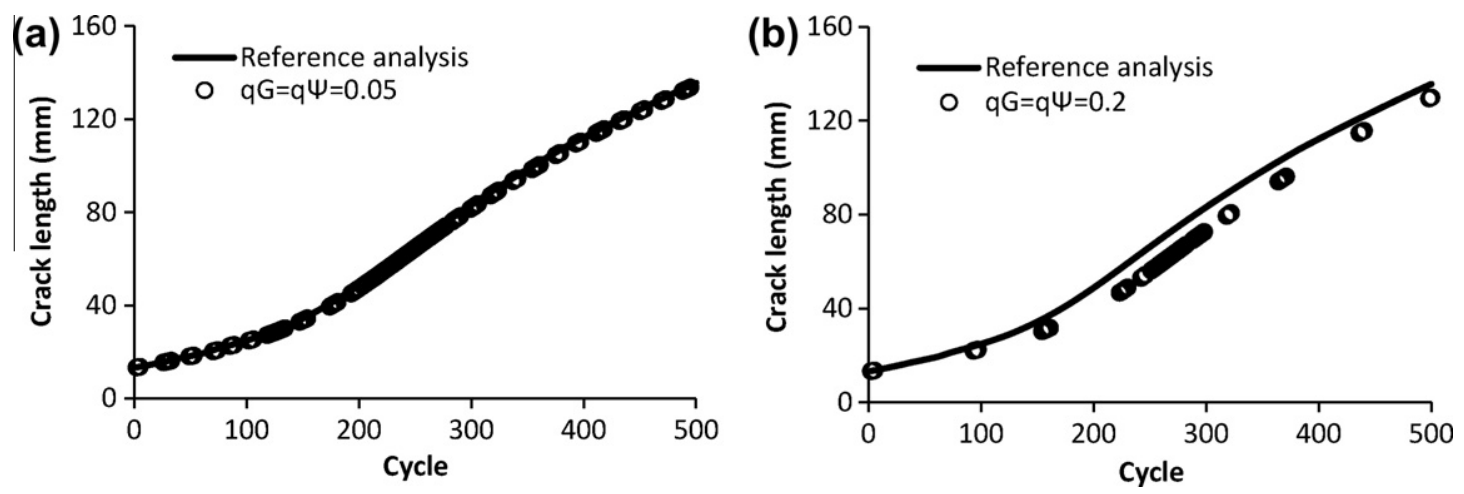

Fig. 8. Crack length vs. number of cycles for (a) control parameter $q_{G}=q_{\Psi}=0.05$ and (b) $q_{G}=q_{\Psi}=0.2$. 
(a)

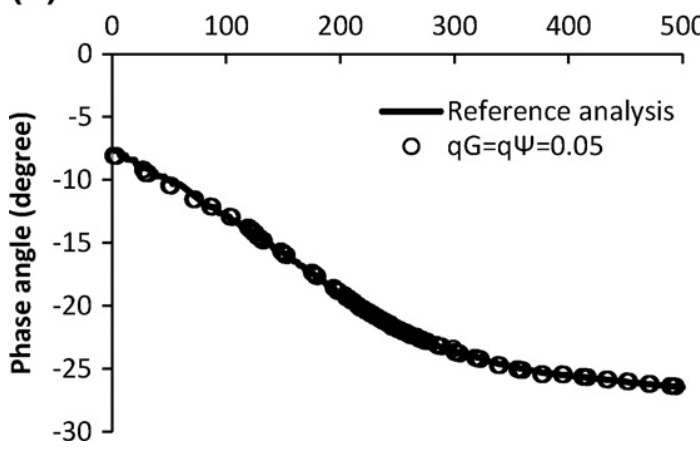

(b)

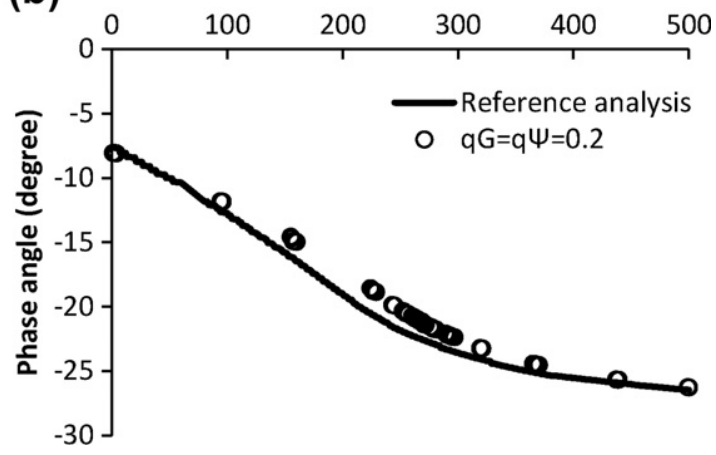

Fig. 9. (a) Mode mixity phase angle vs. number cycles for the reference analysis and the analyses with $q_{G}=q_{\Psi}=0.05$ and $q_{G}=q_{\Psi}=0.2$ control parameters.

Table 2

Number of jumped cycles, computational efficiency, average relative error for $\Delta G$, crack length and phase angle.

\begin{tabular}{|c|c|c|c|c|c|c|}
\hline $\begin{array}{l}\text { Control parameter } \\
q_{G}=q_{\Psi}\end{array}$ & $\begin{array}{l}\text { Number of } \\
\text { simulated cycles }\end{array}$ & $\begin{array}{l}\text { Number of jumps } \\
\text { occurred }\end{array}$ & $R$ & $\begin{array}{l}\text { Average relative error } \\
\text { of } \Delta G(\%)\end{array}$ & $\begin{array}{l}\text { Average relative error of crack } \\
\text { length }(\%)\end{array}$ & $\begin{array}{l}\text { Average relative error of } \\
\text { phase angle (\%) }\end{array}$ \\
\hline 0.025 & 234 & 37 & 0.53 & 1.30 & 0.77 & 0.87 \\
\hline 0.05 & 175 & 25 & 0.65 & 1.39 & 1.06 & 1.22 \\
\hline 0.1 & 115 & 16 & 0.77 & 5.79 & 4.83 & 4.82 \\
\hline 0.2 & 70 & 12 & 0.86 & 5.96 & 7.46 & 5.55 \\
\hline
\end{tabular}

where $N_{\text {jump }}$ is the number of jumped cycles and $N_{\text {ref }}$ is the total number of cycles in the reference analysis. A larger $N$ shows more computational efficiency. To measure the accuracy of the simulations the relative error is defined as [14]:

$E r=\frac{y_{\text {ref }}-y_{\text {jump }}}{y_{\text {ref }}} \times 100$

where $y_{\text {ref }}$ and $y_{\text {jump }}$ are the measured parameters from the reference and cycle jump analysis respectively. The overall average error of the cycle jump method is determined as

$\overline{E r}=\frac{\sum_{N} E r}{N}$

where $N$ is number of simulated cycles and $E r$ is the average error of each cycle. Number of jumped cycles, computational efficiency, average relative error for $\Delta G$, crack length and phase angle for simulations with different control parameters are listed in Table 2 . The computational efficiency of the simulation increases by increasing control parameters, but the accuracy of the simulation decreases. It can be seen that for $q_{G}=q_{\Psi}=0.05$ with a reasonably good accuracy using the cycle jump technique, only 175 cycles are required for the simulation of 500 cycles, resulting in $65 \%$ reduction in the computation time.

\section{Conclusion}

A cycle jump technique for accelerated simulations of fatigue crack growth in a bimaterial interface was presented. The proposed method is based on conducting finite element analysis for a set of cycles to establish a trend line, extrapolating the trend line spanning many cycles, and use the extrapolated state as an initial state for additional finite element simulations. Using the cycle jump technique, fatigue crack growth in the interface of a sandwich beam was simulated for 500 cycles as a numerical example. The computational efficiency and accuracy of the cycle jump technique was discussed and verified based on the three parameters, crack length, difference between maximum and minimum energy release rate in a cycle $(\Delta G)$ and the phase angle against a reference analysis simulating all cycles. The effect of the control parameter governing the cycle jump implementation on the computational efficiency and accuracy was studied.

The results suggest that the computational efficiency of the simulation increases considerably by increasing the control parameter. However the accuracy of the simulation decreases for crack length, $\Delta G$ and phase angle determination. For the control parameter $q_{G}=q_{\Psi}=0.05$ the cycle jump technique requires 175 cycles to simulate 500 cycles, resulting in a $65 \%$ reduction in computation time with a reasonably good accuracy (around $1 \%$ error). The accuracy of quasi-linear problems is less influenced by the control parameter. However, based on the level of nonlinearity of the problem an appropriate control parameter must be chosen. Comparison of the utilized cycle jump method to the other extrapolation methods e.g. Kiewel et al. [12] shows similar computational efficiency and accuracy. However, since the cycle jump method exploits the change in the discrete slope of each state variable increment for the extrapolation, it is believed to be more accurate and computationally effective solution for highly nonlinear problems compared to other methods which exploit only the increment of the variables. This study illustrates that the cycle jump technique is a reliable method to accelerate fatigue crack growth simulation with good accuracy, nonetheless to develop an authentic life prediction method simplified experiments should be conducted to validate and modify the developed scheme.

\section{Acknowledgments}

This work is carried out as an integrated part of the research project "Growth of Debonds in Foam Cored Sandwich Structures under Cyclic Loading" (SANTIGUE) funded by the Danish Research Agency (Grant Nr. 274-05-0324) (R.M. and C.B.) and by funding from the National Science Foundation under Grant CMMI=0825444 (AMK).

\section{References}

[1] Shipsha A, Burman M, Zenkert D. Interfacial fatigue crack growth in foam core sandwich structures. Fatigue Fract Eng Mater Struct 1999;22:123-31.

[2] Quispitupa A, Shafiq B. Fatigue characteristics of foam core sandwich composites. Int J Fatigue 2006;28(1):96-102. 
[3] Berkowitz KC, Johnson W. Fracture and fatigue tests and analysis of composite sandwich structure. J Compos Mater 2005;39(16):1417-31.

[4] Maziere M, Fedelich B. Simulation of fatigue crack growth by crack tip plastic blunting using cohesive zone elements. Procedia Eng 2010;2(1):2055-64.

[5] Kiyak Y, Fedelich B, May T, Pfennig A. Simulation of crack growth under low cycle fatigue at high temperature in a single crystal superalloy. Eng Fract Mech 2008;75(8):2418-43.

[6] Shi Z, Zhang R. Numerical simulation of interfacial crack growth under fatigue load. Fatigue Fract Eng Mater Struct 2009;32:26-32.

[7] Ramanujam N, Vaddadi P, Nakamura T, Singh P. Interlaminar fatigue crack growth of cross-ply composites under thermal cycles. Compos Struct 2008;85:175-87.

[8] Boisse P, Bussy P, Ladeveze P. A new approach in nonlinear mechanics - the large time increment method. Int J Numer Meth Eng 1990;29:647-63.

[9] Cognard JY, Ladeveze P, Talbot P. A large time increment approach for thermomechanical problems. Adv Eng Softw 1999;30:583-93.

[10] Fish J, Yu Q. Computational mechanics of fatigue and life predictions for composite materials and structures. Comput Meth Appl Mech Eng 2002;191:4827-49.

[11] Oskay C, Fish J. Fatigue life prediction using 2-scale temporal asymptotic homogenization. Int J Numer Meth Eng 2004;61:329-59.
[12] Kiewel H, Aktaa J, Munz D. Application of an extrapolation method in thermocyclic failure analysis. Comput Meth Appl Mech Eng 2000;182:55-71.

[13] Van Paepegem W, Degrieck J, De Baets P. Finite element approach for modelling fatigue damage in fibre-reinforced composite materials. Composites Part B - Eng 2001;32:575-88.

[14] Cojocaru D, Karlsson AM. A simple numerical method of cycle jumps for cyclically loaded structures. Int J Fatigue 2006;28:1677-89.

[15] Zenkert D. An introduction to sandwich construction. London: EMAS; 1997.

[16] DIAB. Divinycell H technical data, Laholm. <http://www.diabgroup.com>.

[17] ANSYS. <http://www.ansys.com>.

[18] Berggreen C, Simonsen B, Borum K. Experimental and numerical study of interface crack propagation in foam-cored sandwich beams. J Compos Mater 2007;41:493-520.

[19] Hutchinson JW, Suo Z. Mixed mode cracking in layered materials. Adv App Mech 1992;29:63-191.

[20] Carlsson LA, Karnomateas GA. Structural and failure mechanics of sandwich composites. 1st ed. Springer; 2011 [chapter 9.4].

[21] Karnomateas GA, Pelegri AA, Malik B. Growth of internal delaminations under cyclic compression in composite plates. J Mech Phys Solids 1995;43(6):847-68. 